\title{
EFECTO DE LOS EMBALSES EN EL CONTROL DE AVENIDAS EN EL PIRINEO CENTRAL ESPAÑOL
}

\author{
Juan Ignacio López Moreno, Santiago Beguería y José María García Ruiz \\ Instituto Pirenaico de Ecología, CSIC, Campus de Aula Dei, Apdo 202, 50080 Zaragoza \\ nlopez@ipe.csic.es
}

\begin{abstract}
Resumen: Se estudia el efecto de los embalses en la reducción de la intensidad de avenidas que afectan a los ríos pirenaicos. Se trata de un fenómeno complejo en el que interviene la capacidad de retención de cada embalse, las funciones que desarrollan, el régimen de llenado y vaciado y su ubicación cuando aparecen formando un sistema múltiple. La combinación de estos factores explica los distintos porcentajes de reducción de los caudales más elevados observados en la zona de estudio. A pesar de la existencia de una gran variabilidad de reducción de avenidas, en conjunto los caudales más elevados de los ríos pirenaicos disminuyen de una forma notable, siendo los ríos Aragón y Ésera los que menor control de crecidas experimentan.
\end{abstract}

Palabras clave: Embalses, control de avenidas, gestión de embalses, Pirineo Central español.

\begin{abstract}
The effect of the Pyrenean reservoirs on flood control is studied. It is a complex phenomenon related to the impounded ratio index, the aim of the reservoir, the inflow and outflow regime and the location of the dam when they belong to a multireservoir system. The combination of these factors explains the way in which floods are controlled. In spite of the large variability in the intensity of flood control, a clear decrease of the highest discharges in the Pyrenean rivers has been confirmed. The Aragón and Ésera rivers show the lowest flood control capacity.
\end{abstract}

Key words: Reservoirs, flood control, reservoir management, Central Spanish Pyrenees.

\footnotetext{
* Recibido: 7-04-2003. Aceptado: 9-09-2003.
} 


\section{Introducción}

Los embalses desempeñan una importante función en la regulación de los recursos hídricos, especialmente en ambientes mediterráneos donde la estacionalidad de sus regímenes y la variabilidad interanual de los caudales es muy elevada. La complejidad de funciones que desarrollan (suministro de agua para el riego y abastecimiento urbano e industrial, usos recreativos y control de avenidas) explica el importante incremento en la construcción de presas en las últimas décadas (Takeuchi, 1998).

Generalmente, el control de avenidas no es la función principal para la que se construye un embalse, pero la mayoría de ellos reservan una parte de su gestión a la reducción de los caudales más elevados que reciben. La laminación de las crecidas es un aspecto muy relevante en la gestión de recursos hídricos y planificación del territorio pues, por una parte, permite un rápido llenado del embalse y, por otra, reduce el riesgo de inundación en los sectores localizados aguas abajo de la presa.

Diversos autores han analizado la capacidad de regulación de avenidas por embalses y sus consecuencias. En el Reino Unido se ha estimado que los embalses han reducido las crecidas en torno a un 30\% (Higgs y Petts, 1988). En Austria, caudales que antes de la construcción de centrales hidroeléctricas correspondian a un periodo de retorno de 5 y 50 años actualmente presentan una recurrencia de 75 y 2.500 años respectivamente (Pircher, 1990). En el río Danubio a su paso por Hungría la acción de los embalses ha llevado a un importante descenso de las avenidas de mayor intensidad, mientras pequeñas y medianas crecidas son actualmente más frecuentes (Zsuffa, 1999). Resultados muy similares se han obtenido al estudiar el efecto del sistema de embalses del río Nepean en Australia (Sammut et al., 1995). Otros estudios han analizado cómo ha afectado la reducción de periodos de avenida a la vegetación de ribera y a actividades económicas desarrolladas en la llanura de inundación (Adams et al., 1985; Thomas, 1996), o a la geomorfología de los sectores localizados aguas abajo de la presa (Anders, 2000; Andrews, 1986). En el Pirineo español, se han estudiado los cambios hidrológicos debido al manejo de los embalses instalados en sus principales ríos (López Moreno, 2002), y de una forma más detallada se ha analizado la influencia del embalse de Yesa en la regulación de avenidas, en relación a su régimen de gestión y la capacidad de embalsado que presenta en el momento en que recibe los caudales más elevados (López-Moreno et al., 2002).

A lo largo del siglo XX, la demanda energética y la puesta en marcha de extensas zonas de riego en la Depresión del Ebro explica la construcción de numerosos embalses destinados a regular los abundantes caudales que se generan en el Pirineo Central. Junto a la producción de electricidad y la derivación de agua para la irrigación, la gestión de la mayoría de los embalses pirenaicos ha tenido en cuenta la necesidad de controlar las avenidas. En este trabajo se analiza la intensidad con que los 
embalses reducen la intensidad de las avenidas en el Pirineo Central, y se identifican los factores que explican la capacidad de laminación de los caudales más elevados.

\section{2. Área de estudio}

El área de estudio ocupa gran parte del Pirineo Central español, entre las cuencas altas del río Aragón y río Noguera Pallaresa (Fig. 1). Las máximas altitudes se encuentran en las cabeceras de los ríos Gállego, Cinca, Ésera y Noguera Ribagorzana, donde se superan ocasionalmente los $3000 \mathrm{~m}$ de altitud.

Los ríos pirenaicos cortan de norte a sur un relieve organizado en bandas paralelas, atravesando el sector paleozoico o Pirineo Axial (compuesto por granitos, calizas, pizarras y arcillas), las Sierras Interiores (calizas y areniscas), el Sector del Flysch, la Depresión Interior Altoaragonesa, modelada en margas eocenas, las molasas prepirenaicas (areniscas y arcillas) y las Sierras Exteriores (calizas y margas) que constituyen el extremo sur de la zona de estudio antes de alcanzar la Depresión del Ebro. Las presas de los embalses pirenaicos tienden a estar apoyadas sobre los materiales más consistentes de las Sierras Interiores, en la Depresión Interior e inmediatamente al norte de las Sierras Exteriores, aprovechando pequeñas depresiones margosas.

El clima tiende a perder sus características oceánicas en sentido norte sur y de oeste a este, adquiriendo progresivamente rasgos más mediterráneo-montanos con tendencia continental. Este cambio supone un descenso de la precipitación y un incremento de las temperaturas, si bien estas últimas están controladas principalmente por la altitud. La precipitación oscila entre más de $2000 \mathrm{~mm}$ anuales por encima de $2000 \mathrm{~m}$ de altitud y poco más de $500 \mathrm{~mm}$ al sur de la zona de estudio. Las precipitaciones más abundante suceden entre octubre y junio, con un ligero descenso en enero y febrero, excepto en los ambientes más oceánicos, donde el máximo anual se concentra en invierno. Los eventos de precipitación muy intensos pueden producirse a lo largo de todo el año (White et al., 1997; García Ruiz et al., 2001) aunque son más frecuentes en otoño.

Desde diciembre hasta marzo la isoterma de $0^{\circ} \mathrm{C}$ se encuentra en torno a 1600 m.s.n.m. (García Ruiz et al., 1985), por lo que la acumulación y la fusión de la nieve tienen un importante papel en el régimen fluvial. Su influencia es mayor hacia el este debido a la mayor altitud media de las cabeceras y a la mayor superficie ocupada por áreas situadas a más de 2000 m s.n.m, especialmente entre los ríos Gállego y Noguera Ribagorzana (García Ruiz et al., 1980). De ahí que el periodo de aguas altas primaverales se desplace desde abril y mayo en la cabecera del río Aragón, hasta junio en los ríos Ara, Cinca, Ésera y Noguera Ribagorzana. El periodo de aguas bajas es claramente estival al oeste e invernal al este (García Ruiz et al., 2001). 


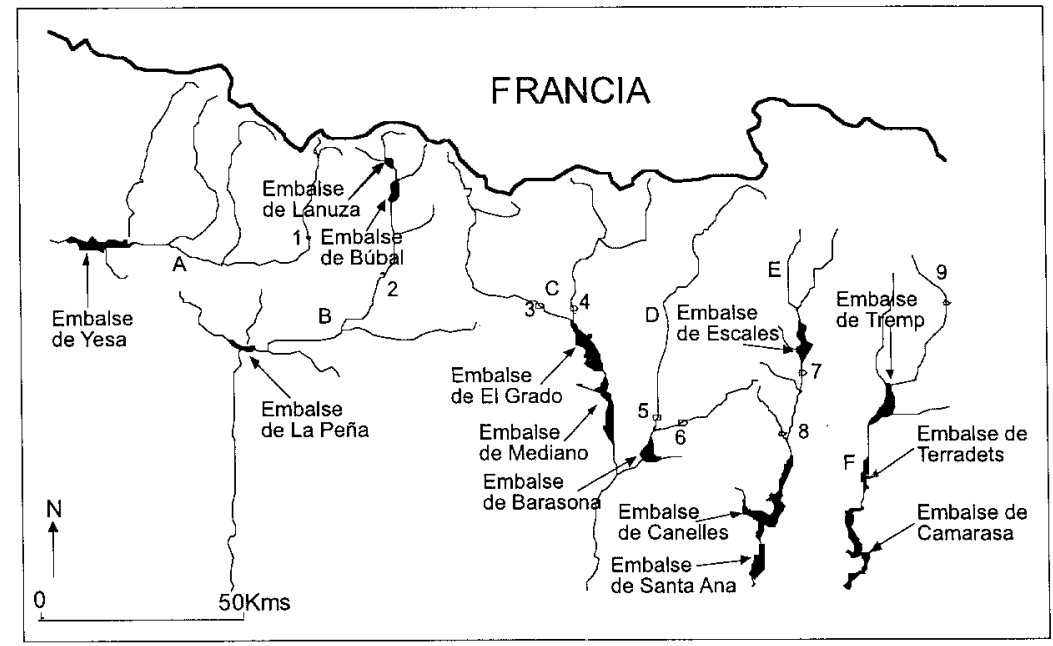

Figura 1. Área de estudio.

A: Río Aragón; B: Río Gállego; C: Río Cinca; D: Río Ésera; E: Río Noguera Ribagorzana; F: Río Noguera Pallaresa. 1: Jaca; 2: Sabiñánigo; 3: Boitaña; 4: Ainsa; 5: Graus; 6: Capella; 7: Pont de Suert; 8: Puente de Montañana; 9: Sort.

\section{Material y métodos}

Los datos utilizados han sido facilitados por la Confederación Hidrográfica del Ebro, disponiéndose de valores diarios del volumen almacenado y del caudal a la salida del embalse. De los embalses que cuentan con un canal de derivación se dispone de la información de sus salidas totales, y del caudal derivado hacia el canal. El caudal que circula por el río aguas abajo del embalse se obtiene restando a las salidas totales el agua derivada hacia el canal.

El caudal de entrada se ha obtenido sumando las salidas del embalse a la variación diaria del volumen embalsado. La obtención de la aportación al embalse presenta dos problemas que limitan la fiabilidad de los datos: i) la acumulación de sedimento en el vaso del embalse supone un cambio en la relación altura de la lámina de agua con el volumen embalsado, por lo que se requiere cambiar la ecuación que establece dicha relación mediante la realización de batimetrías de forma periódica. El CEDEX (Centro de Estudios y Experimentaciones) lleva a cabo campañas batimétricas anuales, pero en el Pirineo han sido muy pocas las realizadas, continuando la mayor parte de los embalses estudiados sin corregir desde la fecha de su construcción; ii) pequeños errores en la estimación de la altura de la lámina de agua suponen un importante error en la estimación del volumen embalsado, lo que implica un 
error en el calculo del caudal de entrada al embalse. Los citados errores han llevado en ocasiones a utilizar como caudales de entrada los valores de caudal registrados en estaciones de aforo cercanas a la cola del embalse. Asi se ha hecho en el embalse de Yesa (suma de los ríos Aragón en la cola del embalse y Esca en Sigüés) y en el de Barasona (suma de los ríos Ésera en Graus e Isábena en Capella).

Al no tratarse, en general, de aforos instalados en cursos naturales, los datos no se pueden comparar con otras series cercanas o regionales, como se hace con los datos climáticos o de aforo. Este hecho dificulta el relleno de datos ausentes y la aceptación o rechazo de datos anómalos, pues en numerosas ocasiones no se puede distinguir si nos encontramos ante un error instrumental o de simple trascripción a formato digital, o son fruto de la actuación de sus gestores (vaciado repentino del embalse, laminación o generación de una crecida, tareas de limpieza en canales o compuertas, etc.).

Una vez realizado un análisis de calidad, se ha procurado utilizar la mayor cantidad de datos disponibles para cada uno de los embalses estudiados. En aquellos ríos sobre los que se han instalado más de un embalse, se ha elegido el máximo periodo disponible común a todos los embalses que conforman el sistema (Tabla 1).

Se ha estudiado el efecto de control de crecidas de cada embalse comparando el caudal estimado para distintos periodos de retorno aguas arriba y abajo de la presa. No se

Tabla 1. Características de los embalses estudiados.

\begin{tabular}{|c|c|c|c|c|c|c|c|c|}
\hline Enbalse & Río & $\begin{array}{l}\text { Año de } \\
\text { constritcció }\end{array}$ & $\begin{array}{l}\text { Periodo } \\
\text { in estudiado }\end{array}$ & $\begin{array}{l}\text { Capacidad } \\
\text { (hombi) }\end{array}$ & $\begin{array}{l}\text { Aportación } \\
\text { media anual } \\
\left(\mathrm{hmin}^{3}\right)\end{array}$ & $\begin{array}{l}\text { Ratio } \\
\text { de } \\
\text { retención }\end{array}$ & $\mathrm{U}_{S_{3}}$ & $\begin{array}{l}\text { Alitind } \\
\text { (m.sn.m. })\end{array}$ \\
\hline Yesa & Aragón & 1959 & $1959-2001$ & 446,89 & 1327,74 & 0,34 & Regadio & 489 \\
\hline Lanuza & Gállego & 1980 & $1981-2000$ & 16,86 & 262,07 & 0,06 & Hidroeléctrico & 1283 \\
\hline Búbal & Gállego & 1971 & $1981-2000$ & 64,26 & 429,29 & 0,15 & Hidroeléctrico & 1080 \\
\hline * Sistema Galllego (Lamuza+Bubal) & Gállego & - & $1981-2000$ & 81,12 & 429,29 & 0,19 & Hidroeléctrico & $1283-1080$ \\
\hline La Pena & Gállego & 1913 & $1954-2001$ & 15,44 & 610,00 & 0,03 & Regadío & 536 \\
\hline Mediano & Cinca & 1973 & $1988-2000$ & 436,35 & 1293,06 & 0,34 & Hidroeléctrico & 528 \\
\hline El Grado & Cinca & 1969 & $1988-2000$ & 399,45 & 1446,46 & 0,28 & Hidroeléctrico / Regadío & 450 \\
\hline * Sistema Cinca & Cinca & - & $1988-2000$ & 835,80 & 1446,46 & 0,58 & Hidroeléctrico / Regadio & $528-450$ \\
\hline Barasona & Ésera & 1932 & $1958-2000$ & 92,20 & 833,36 & 0,11 & Hidroeléctrico / Regadío & 441 \\
\hline Escales & Noguera Ribagorzana & 1955 & $1990-2000$ & 152,36 & 566,86 & 0,27 & Hidroeléctrico & 900 \\
\hline Canelles & Noguera Ribagorzana & 1957 & $1990-2000$ & 678,00 & 662,83 & 1,02 & Hidroeléctrico & 506 \\
\hline Santa Ana & Noguera Ribagorzana & 1961 & $1990-2000$ & 237,00 & 684,03 & 0,35 & Hidroeléctrico / Regadio & 375 \\
\hline * Sistema Noguera Ribagorzana & Noguera Ribagorzana & - & $1990-2000$ & 1067,36 & 684,03 & 1,56 & Hidroeléctrico / Regadio & $900-375$ \\
\hline Tremp & Noguera Pallaresa & 1916 & $1958-2000$ & 257,80 & 1136,82 & 0,23 & Hidroeléctuico & 541 \\
\hline Terradets & Noguera Pallaresa & 1935 & $1958-2000$ & 33,20 & 1218,90 & 0,03 & Hidroeléctrico & 412 \\
\hline Camarasa & Noguera Pallaresa & 1920 & $1958-2000$ & 163,39 & 1302,16 & 0,13 & Hidroeléctrico & 376 \\
\hline * Sistema Pallaresa & Noguera Pallaresa & $\cdot$ & $1958-2000$ & 454,39 & 1302,16 & 0,35 & Hidroeléctrico & $541-346$ \\
\hline
\end{tabular}


ha realizado una comparación de la intensidad de las avenidas entre el periodo anterior y posterior a la construcción del embalse, pues en ocasiones no se cuenta con series de caudal lo suficientemente largas; además, cambios en usos de suelo y del tipo de cubierta vegetal de las cuencas drenadas, junto a la variabilidad climática, han podido producir cambios en la intensidad de las crecidas (López Moreno et al., en prensa) y por tanto dificultar el estudio del efecto de los embalses en la laminación de avenidas.

Los caudales correspondientes a distintos periodos de retorno se han estimado ajustando las series de excedencia que superan el centil 97 a una distribución exponencial. La utilización de series de excedencia, frente al uso de series de máximos anuales, permite un uso más eficiente de la información contenida en las series originales, pues permite incluir más de un evento anual si cumplen los requisitos para ser considerados extremos (Cunnane et al., 1973, Madisen et al., 1997). Además, uno de los mayores problemas que se asocia al uso de series de excedencia, como es la dificultad para garantizar la independencia de las observaciones, no afecta significativamente a los resultados (Beguería, 2002). La elección de la distribución de frecuencias Exponencial, frente a otras posibilidades, se ha basado en el buen ajuste de las muestras utilizadas con esta distribución en la gráfica de L momentos (1993, Hosking, 1990; Vogel et al., 1993). Para calcular el caudal correspondiente a distintos periodos de retorno, las muestras se ajustan a la distribución Exponencial mediante el cálculo de los parámetros origen y escala (Rao et al., 2000). Se ha rechazado el uso de distribuciones triparamétricas (por ej., General Pareto), que incluyen un tercer parámetro de forma, pues este último está sujeto a un gran margen de error (Beguería et al. 2000). En este estudio el posible error en el cálculo del parámetro de forma es mayor, pues los datos están condicionados por la gestión humana y algunas series apenas superan los diez años de longitud.

Los parámetros necesarios para el ajuste de los datos observados se han obtenido a partir de L-momentos, que sustituyen al uso de momentos normales de una forma ventajosa. Los L-momentos se obtienen por combinación lineal de los momentos ponderados por probabilidad (PWM) (Greenwood et al., 1979). La mayor ventaja del uso de L-momentos es que éstos no se encuentran influidos por la existencia de elementos extremos, como sucede con los momentos ordinarios (media y desviación estándar), por lo que son mucho más fiables.

\section{Características de los embalses estudiados}

La función en la regulación de las crecidas ha sido estudiada en todos los embalses de la zona de estudio que cuentan con una capacidad de embalsado superior a $15 \mathrm{hm}^{3}$. La mayoría de los ríos pirenaicos se encuentran regulados por sistemas de 
embalses como sucede en los ríos Gállego, Cinca, Noguera Ribagorzana y Noguera Pallaresa. Tan sólo los ríos Aragón y Ésera se encuentran regulados por un embalse principal y pequeños embalses de cabecera destinados a la producción hidroeléctrica.

La Tabla 1 muestra las principales caracteristicas de los embalses estudiados: capacidad de embalsado, destino principal de las aguas y ratio de retención calculado para cada uno de los embalses y sistema de embalses. El uso fundamental de los embalses de Yesa y La Peña es el suministro de agua para regadío. El Grado, Santa Ana y Barasona combinan la producción hidroeléctrica con el suministro de agua para usos agrícolas. El resto de los embalses se destinan fundamentalmente a la producción hidroeléctrica.

La capacidad de los embalses estudiados oscila entre 15 y $678 \mathrm{hm}^{3}$. Los situados a mayor altitud, en las zonas de cabecera, poseen generalmente un tamaño reducido (hasta $64 \mathrm{hm}^{3}$ ) y están destinados a la producción hidroeléctrica. Los embalses con mayor capacidad se encuentran ubicados sobre litologías blandas inmediatamente al norte de las Sierras Exteriores. Entre ellos destacan Canelles $\left(678 \mathrm{hm}^{3}\right)$, Yesa $(447$ $\mathrm{hm}^{3}$ ) y Mediano $\left(436 \mathrm{hm}^{3}\right)$.

La relación entre el caudal que llega a un embalse o sistema de embalses y su capacidad es la ratio de retención. El embalse de Canelles es el que presenta una mayor ratio de retención, hasta el punto de que su capacidad de almacenamiento es mayor que el promedio anual de aportación que recibe. La capacidad de almacenamiento de otros embalses como Yesa, Mediano, El Grado, Santa Ana y Escales, equivale a un $25 \%$ de su caudal. Finalmente hay embalses con una capacidad muy limitada de regulación. Las ratios de retención más bajos se observan en embalses de poca capacidad situados en los sectores inferiores de la zona de estudio como el de La Peña y Terradets. El río Noguera Ribagorzana es el que, en conjunto, con su sistema de tres embalses, presenta mayor ratio de retención (1.56). Los ríos Cinca, Noguera Pallaresa y Aragón muestran ratios intermedios, con valores de $0.58,0.35 \mathrm{y}$ 0.34 respectivamente. Los ríos Ésera y Gállego son los que poseen una menor ratio de retención, con valores que no superan 0.19 .

\section{Resultados}

\subsection{Efecto de los embalses en la laminación de avenidas}

Los ríos estudiados pueden registrar crecidas de gran envergadura (Martí Bono y Puigdefábregas, 1983; García Ruiz et al., 1983 y 2001). La Tabla 2 muestra el caudal medio de los ríos a la entrada de cada embalse y el número por el que se multiplica 
durante avenidas correspondientes a un periodo de retorno estimado de 5 y 50 años. El hecho de que la longitud de la serie de cada embalse sea distinta dificulta la comparación entre los distintos ríos, pues cambia tanto el número como las características de los eventos estudiados, lo que supone cambios importantes en la estimación de caudales con una determinada recurrencia. No obstante, permite apreciar que los ríos pirenaicos en momentos de avenida pueden multiplicar de forma muy notable su caudal. Entre todos destacan los ríos Gállego y Ésera a la entrada de los embalses de La Peña y Barasona, que multiplican su caudal medio por más de quince y veinte veces en avenidas de 5 y 50 años de recurrencia respectivamente. Es importante tener en cuenta, no obstante, que el río Gállego en cabecera (Lanuza y Búbal) muestra un comportamiento muy poco torrencial, y que, por lo tanto, es a partir de Búbal cuando se incrementa notablemente la intensidad de sus avenidas. Otros ríos, como el Noguera Pallaresa, aun contando con series largas (Tabla 1), presentan un incremento más moderado de su caudal medio, quedando patente la existencia de importantes contrastes en la intensidad de las avenidas de los ríos estudiados.

Tabla 2. Número de veces que exceden el caudal medio anual los caudales estimados a la entrada de los embalses, para un periodo de retorno de 5 y 50 años.

\begin{tabular}{|lccc|}
\hline Embalse & Qm $\left(\mathrm{m}^{3} \mathrm{~s}^{-1}\right)$ & Q5 & Q50 \\
\hline Yesa & 42,0 & 13,2 & 18,5 \\
Lanuza & 8,3 & 5,5 & 6,9 \\
Búbal & 13,6 & 7,1 & 9,6 \\
La Peña & 18,6 & 17,0 & 24,8 \\
Mediano & 41,1 & 13,8 & 19,7 \\
El Grado & 45,2 & 7,5 & 10,7 \\
Barasona & 25,9 & 16,4 & 24,0 \\
Escales & 17,9 & 7,3 & 10,1 \\
Canelles & 20,5 & 8,6 & 12,2 \\
Santa Ana & 20,8 & 4,4 & 5,4 \\
Tremp & 36,4 & 10,0 & 13,9 \\
Terradets & 38,0 & 6,6 & 8,8 \\
Camarasa & 41,6 & 7,0 & 9,6 \\
\hline
\end{tabular}

La Tabla 3 muestra los caudales estimados para un periodo de retorno de 5 y 50 años a la entrada y salida de cada embalse, y la reducción que representan (en tanto por ciento) las avenidas aguas abajo de la presa. Los resultados obtenidos muestran valores muy desiguales. Los embalses de Canelles, Mediano y Santa Ana presentan un porcentaje de reducción aguas abajo de la presa cercano al 50\%. La capacidad de control de crecidas es menor en otros embalses, como Búbal, Tremp y Escales; aun así, los caudales máximos esperados en 5 y 50 años experimentan un descenso cercano al 30\%. Los embalses de Lanuza, Yesa y Barasona reducen ese porcentaje a una cifra próxima al 15 
$\%$ en los dos primeros casos y apenas superando el 5\% en el último. Los embalses de Terradets, Camarasa, El Grado y La Peña, ejercen un papel más limitado en el control de avenidas, hasta el punto de que los caudales estimados aguas abajo de la presa pueden llegar a ser superiores a los que llegan al embalse en determinados eventos. El porcentaje de reducción es, naturalmente, un indicador a efectos comparativos de la capacidad de respuesta de los embalses frente a las avenidas. Pero no es, ni mucho menos, una situación media o habitual. Los datos se refieren al pico de caudal máximo esperable para dos periodos de retorno, 5 y 50 años, pero eso no significa que los embalses de El Grado, La Peña o Terradets, por ejemplo, no ejercen apenas control sobre las avenidas.

Tabla 3. Reducción de las avenidas aguas abajo de los embalses estudiados

\begin{tabular}{|l|c|c|c|c|c|c|}
\hline & \multicolumn{3}{|c|}{ Periodo de retorno: 5 años } & \multicolumn{3}{c|}{ Periodo de retorno: 50 años } \\
\hline Embalse & Entrada $\left(\mathrm{m}^{3} \mathrm{~s}^{-1}\right)$ & Salida $\left(\mathrm{m}^{3} \mathrm{~s}^{-1}\right)$ & Reducción $\%$ & Entrada $\left(\mathrm{m}^{3} \mathrm{~s}^{-1}\right)$ & Salida $\left(\mathrm{m}^{3} \mathrm{~s}^{-1}\right)$ & Reducción \% \\
\hline Yesa & 554 & 460 & 17 & 779 & 650 & 17 \\
Lanuza & 46 & 39 & 15 & 57 & 47 & 18 \\
Búbal & 97 & 63 & 35 & 131 & 81 & 38 \\
La Peña & 317 & 322 & -2 & 462 & 471 & -2 \\
Mediano & 568 & 300 & 47 & 811 & 417 & 49 \\
El Grado & 337 & 342 & -1 & 484 & 481 & 1 \\
Barasona & 426 & 397 & 7 & 621 & 587 & 5 \\
Escales & 131 & 97 & 26 & 181 & 129 & 29 \\
Canelles & 176 & 87 & 51 & 250 & 107 & 57 \\
Santa Ana & 91 & 53 & 42 & 112 & 60 & 46 \\
Tremp & 364 & 250 & 31 & 507 & 343 & 32 \\
Terradets & 250 & 243 & 3 & 336 & 326 & 3 \\
Camarasa & 293 & 289 & 1 & 401 & 402 & 0 \\
\hline
\end{tabular}

Lo que queda claro a partir de la Tabla 3 es que hay sistemas de embalses con una fuerte reducción de las avenidas. Ése es el caso de los embalses del río Noguera Ribagorzana, que pueden llegar a rebajar, en conjunto, en un $80 \%$ el pico de crecida esperable para un periodo de retorno de 50 años. En el caso del río Cinca ese porcentaje se reduce al $45 \%$, especialmente por el embalse de Mediano más que por El Grado. Las avenidas en la cabecera del río Gállego, regulada por los embalses de Lanuza y Búbal, también experimentan una fuerte reducción. En el caso del río Ésera, en cambio, la reducción del pico de crecida es mucho más moderada.

\subsection{Factores que condicionan la capacidad de control de avenidas}

La Tabla 3 ha demostrado que existen importantes diferencias en la respuesta de los embalses frente a las avenidas. A continuación se consideran las características de cada 
embalse y el régimen de explotación al que están sometidos, considerados como los factores que explican gran parte de la variabilidad obtenida. De todos ellos, el de Canelles muestra un comportamiento alejado de la mayoría de los análisis realizados. Por esta razón ha sido excluido de la estimación de la correlación entre la reducción de avenidas y las distintas variables analizadas, aunque se representa en los gráficos.
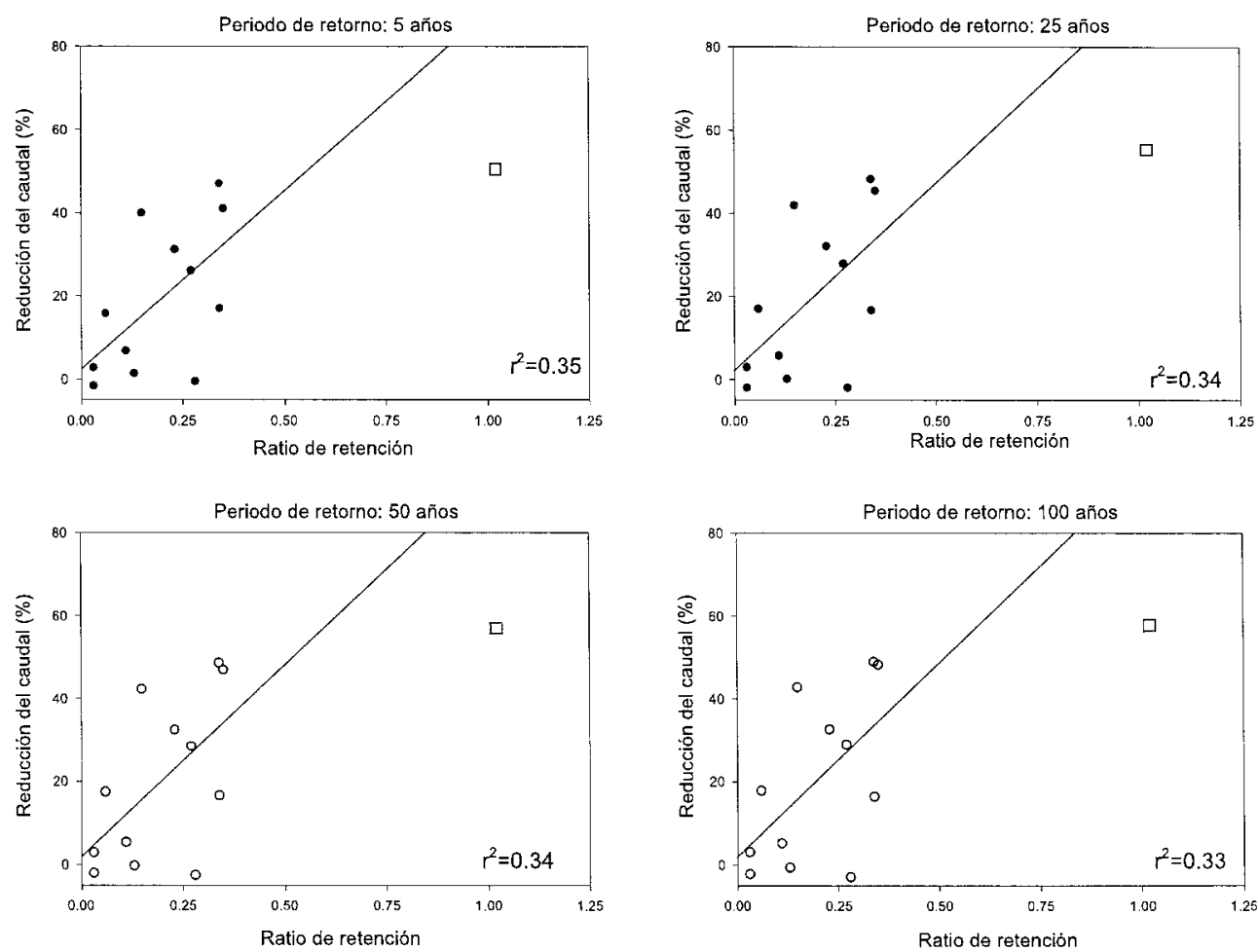

․ Embalse de Canelles

Figura 2. Relación entre la ratio de retención y la reducción de avenidas para distintos periodos de retorno.

La Fig. 2 muestra la relación entre la ratio de retención de cada embalse y la reducción que experimentan las avenidas correspondientes a distintos periodos de recurrencia. Como cabría esperar, los embalses con mayor ratio de retención poseen una mayor capacidad de laminación. De hecho, el coeficiente de correlación de Pearson oscila entre 0.33 y 0.35 . Sin embargo, la dispersión de los puntos alrededor de la recta 
de regresión es muy grande, lo que sugiere que una parte importante de la variabilidad en la capacidad de laminación de crecidas no queda explicada por su ratio de retención. La posición del embalse de Canelles respecto a los demás confirma su especial comportamiento, con una laminación de avenidas inferior a lo que cabría esperar por su elevado ratio de retención. Su respuesta frente a las avenidas muy probablemente se debe a que durante determinados eventos se realiza un desembalse relativamente elevado que es fácilmente controlado por el embalse de Santa Ana, situado inmediatamente aguas abajo de su presa. De esta forma, se acelera el llenado de este último, sin suponer riesgo alguno de inundación.
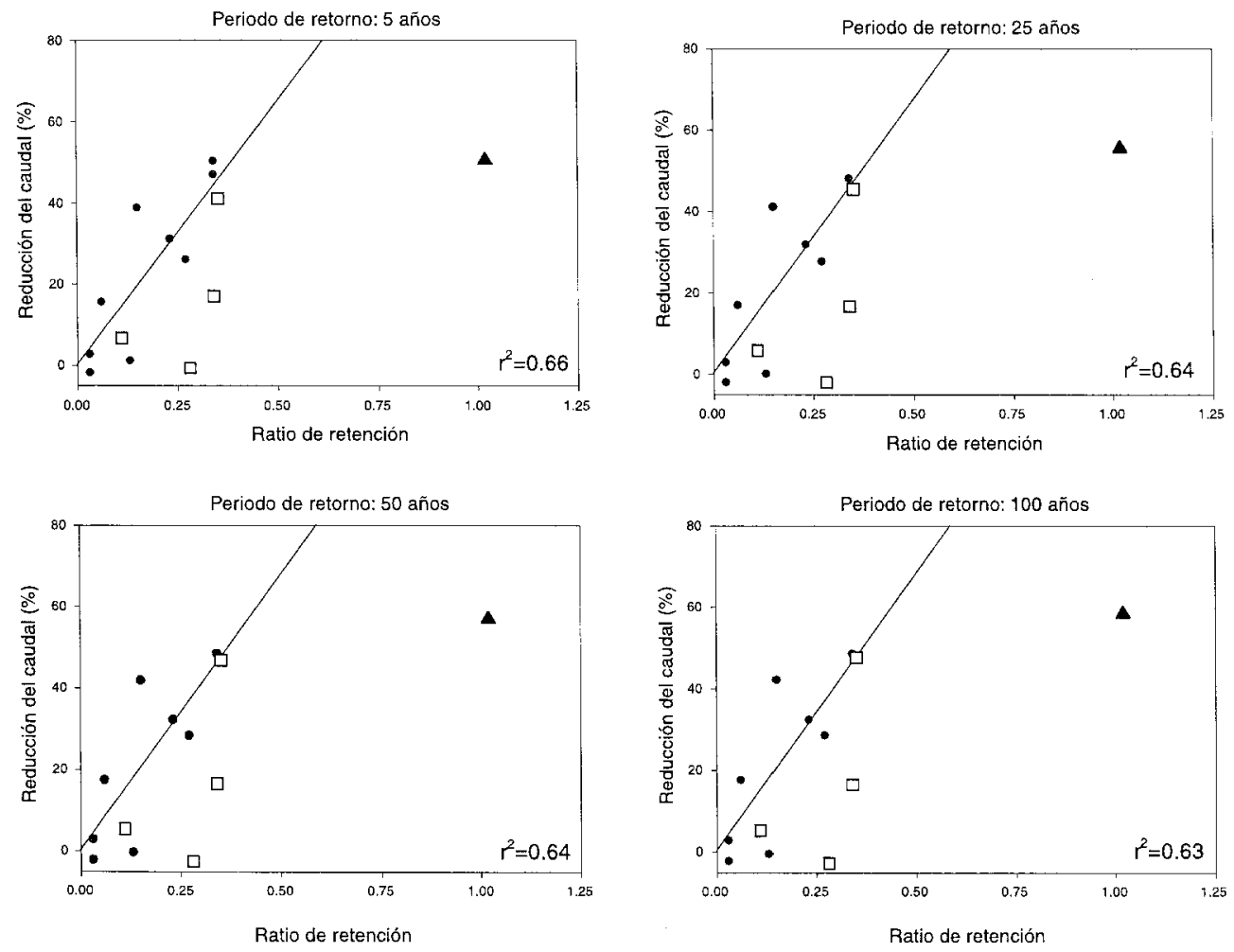

- Hidroeléctrico

․ Hidroeléctrico/irrigación

\section{- Embalse de Canelles}

Figura 3. Relación entre ratio de retención y reducción de avenidas para distintos periodos de retorno, distinguiendo la función que desempeñan los embalses. El coeficiente de determinación se refiere a los embalses hidroeléctricos. 
Si se diferencia el uso que recibe cada embalse (Fig. 3), la ratio de retención explica una mayor proporción de la variabilidad. Considerando, únicamente los embalses destinados a la producción hidroeléctrica, los coeficientes de correlación oscilan entre 0.66 y 0.63 . Es interesante señalar que de los cuatro embalses destinados al suministro de agua para la agricultura, o que combinan esta labor con la producción hidroeléctrica, tres se encuentran claramente por debajo de la tendencia general. La Fig. 4 ayuda a interpretar esta cuestión. Así, el embalse de Yesa posee un nivel de embalsado relativamente bajo en otoño, con progresivo incremento hasta estabilizarse en invierno, periodo en el que se deja un margen de seguridad elevado para laminar las crecidas de la estación fría del río Aragón y reducir sus efectos en el río Ebro (López Moreno et al., 2000). A partir de marzo el embalse tiende a aumentar de nuevo el agua almacenada, hasta alcanzar el máximo en mayo-junio. De esa forma se encuentra en condiciones óptimas de atender a la demanda de agua para riego. En cambio, se reducen mucho sus posibilidades de laminación de avenidas durante la segunda mitad de la primavera.

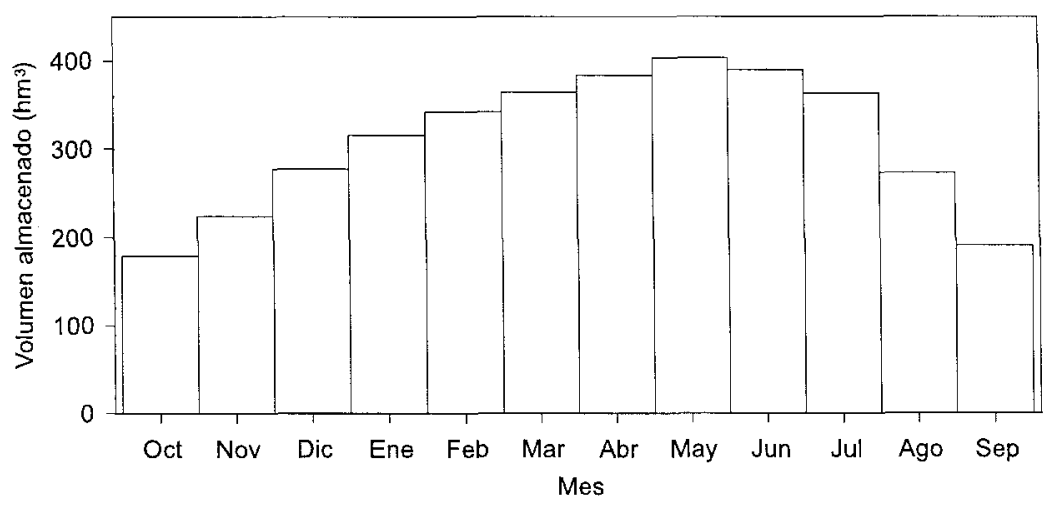

Figura 4. Evolución del nivel de agua almacenada en el embalse de Yesa.

La Tabla 4 muestra las diferencias en la capacidad para laminar avenidas según se encuentre el embalse por encima o por debajo del $70 \%$ de su nivel máximo. Resulta evidente que el nivel de llenado del embalse debe explicar gran parte de la respuesta frente a las avenidas. Sin embargo, la reducción de caudales en la Tabla 4 muestra una gran variabilidad. Si el embalse está con un nivel de agua almacenada inferior al $70 \%$ lo normal es que se produzca una notable laminación de la crecida. Así sucede en los embalses de Yesa, Lanuza, Búbal, Mediano, Escales, Santa Ana y Tremp, incluso si se trata de avenidas correspondientes a un periodo de retorno de 50 años. Pero otros embalses producen una laminación muy inferior (La Peña y Barasona, por 
su reducido tamaño). Si el embalse se encuentra por encima del $70 \%$ de su capacidad, sólo Mediano, Santa Ana y Tremp tienen un importante efecto de laminación; el resto de los embalses desalojan casi todo el caudal que entra, incluso para una avenida de 5 años de recurrencia. En estos casos cuando el embalse supera un determinado umbral de volumen almacenado, tienden a desembalsar buena parte de los caudales que llegan, bien porque su capacidad de retención es ya muy limitada, o bien porque prefieren mantener un margen de seguridad en previsión de futuras avenidas. En ocasiones si el embalse se encuentra muy cerca de su máxima capacidad, puede llegar a incrementarse la intensidad de la avenida que se produce aguas debajo de la presa. Un ejemplo de esta situación se produjo en el embalse de Yesa en noviembre de 1969 , cuando se sucedieron cinco días con caudales de entrada que excedían los $300 \mathrm{~m}^{3} \mathrm{~s}^{-1}$, lo que llevó al embalse al $95 \%$ de su capacidad. El incremento posterior de la avenida hasta $527 \mathrm{~m}^{3} \mathrm{~s}^{-1}$ provocó un desembalse que alcanzó los $1324 \mathrm{~m}^{3} \mathrm{~s}^{-1}$. Durante la crecida de noviembre de 1982 el río Gállego también experimentó una situación similar. Así, tras cuatro días en los que el embalse de Búbal recibió caudales que llegaron a $155 \mathrm{~m}^{3} \mathrm{~s}^{-1}$, al alcanzar un volumen almacenado próximo al $90 \%$ de su capacidad se produjo un desembalse que superó los $185 \mathrm{~m}^{3} \mathrm{~s}^{-1}$. Aguas abajo, durante esta misma crecida, el río Gállego tuvo un hidrograma de crecida con un tramo ascendente mucho más brusco a la salida del embalse de la Peña que en Anzánigo, y un pico de crecida ligeramente superior (García Ruiz et al., 1983).

Tabla 4. Reducción de avenidas aguas debajo de los embalses distinguiendo dos niveles de embalsado.

\begin{tabular}{|l|c|c|c|c|}
\hline \multirow{2}{*}{ Embalse } & \multicolumn{2}{|c|}{ Recurrencia: 5 años } & \multicolumn{2}{c|}{ Recurrencia: 50 años } \\
\cline { 2 - 5 } & $0-70 \%$ & $70-100 \%$ & $0-70 \%$ & $70-100 \%$ \\
\hline Yesa & 81 & 6 & 82 & 6 \\
Lanuza & 87 & 2 & 91 & -1 \\
Bubal & 87 & 12 & 91 & 14 \\
La Peña & 37 & 0 & 39 & 0 \\
Mediano & 62 & 25 & 67 & 23 \\
El Grado & 47 & 5 & 44 & 2 \\
Barasona & 29 & 5 & 29 & 4 \\
Escales & 68 & 0 & 73 & -1 \\
Canelles & 44 & 10 & 51 & 11 \\
Santa Ana & 61 & 33 & 64 & 30 \\
Tremp & 65 & 18 & 70 & 22 \\
Terradets & 31 & 1 & 31 & -1 \\
Camarasa & 36 & 1 & 43 & 0 \\
\hline
\end{tabular}

A partir de la Tabla 4 puede deducirse que en el río Aragón el embalse de Yesa tiene escasa capacidad de regulación de avenidas cuando se encuentra casi lleno, lo 
que ocurre en primavera. En el río Gállego la laminación de avenidas de cabecera depende sustancialmente del embalse de Búbal y, aguas abajo, el embalse de La Peña deja pasar casi todas las avenidas de primavera. En el río Cinca, la Figura 5 demuestra que el embalse de Mediano controla de forma muy notable las crecidas, disminuyendo en torno a un $50 \%$ los caudales esperados para distintos periodos de retorno; en cambio, en el embalse de El Grado los caudales predichos aguas arriba y abajo son muy similares, debido a la labor previa de laminación que efectúa el embalse de Mediano. Una gestión de avenidas muy similar se lleva a cabo en el sistema múltiple instalado en el río Noguera Pallaresa.
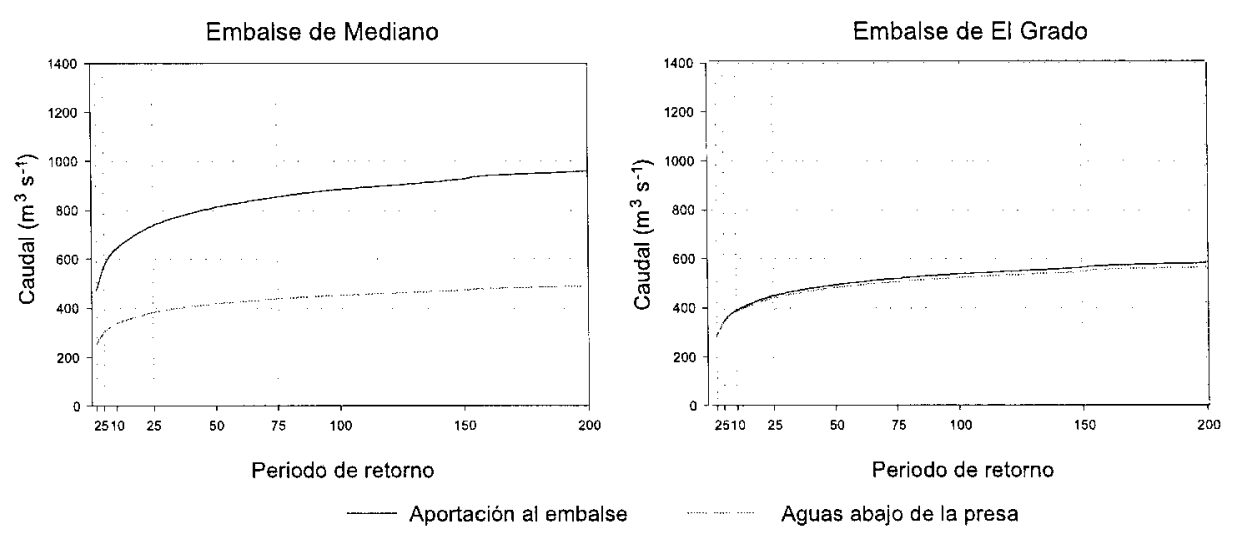

Figura 5. Caudales estimados para distintos periodos de retorno a la entrada y aguas abajo del sistema de embalses instalado en el río Cinca.

En el sistema múltiple de embalses del río Noguera Ribagorzana (Fig. 6), el control de las crecidas se reparte entre los tres embalses. El embalse de Escales, en la cabecera, presenta un valor medio de retención de avenidas cercano al $25 \%$, dejando pasar caudales elevados que serán utilizados para el llenado de los embalses situados aguas abajo. El embalse de Canelles es el que más regula las avenidas reduciendo sus caudales en más de un $50 \%$, debido a que se trata de un embalse con una ratio de retención muy elevado. Finalmente, el embalse de Santa Ana recibe caudales ya muy regulados, que raras veces superan los $100 \mathrm{~m}^{3} \mathrm{~s}^{-1}$. A pesar de ello es capaz de reducir los picos de avenida en más de un 40\%. De este modo el efecto acumulado de los tres embalses conduce a la desaparición de caudales elevados aguas abajo del sistema. 

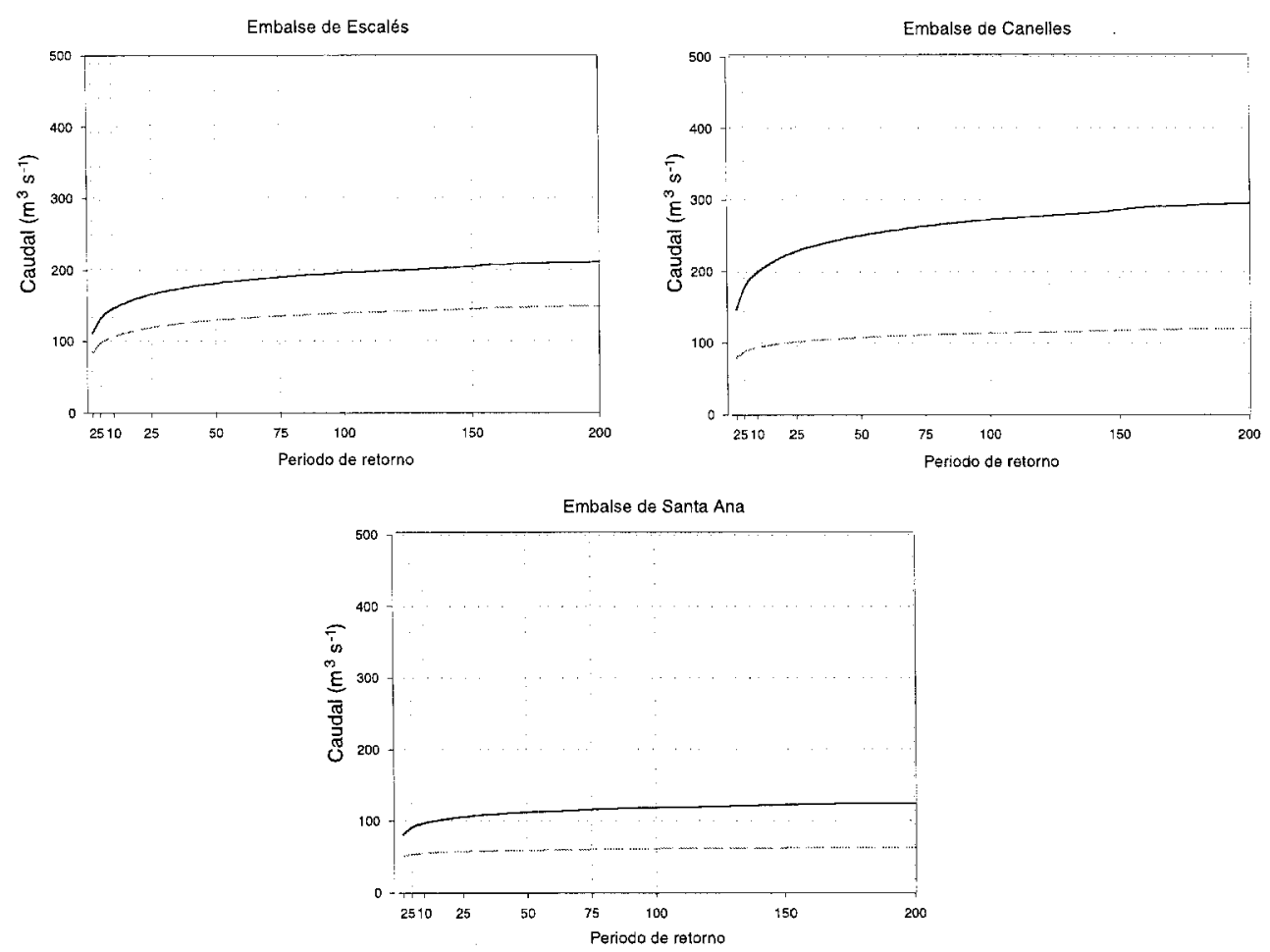

- Aportación al embalse _....... Aguas abajo de la presa

Figura 6. Caudales estimados para distintos periodos de retorno a la entrada y aguas abajo de las presas del sistema de embalses instalado en el río Noguera Ribagorzana.

\section{Conclusiones}

Los embalses pirenaicos tienen como objetivo prioritario la acumulación de grandes volúmenes de agua para el abastecimiento de perímetros de riego (Bardenas, Riegos del Alto Aragón y Aragón y Cataluña) y para producción hidroeléctrica (cabecera del río Gállego, complejo de Mediano y El Grado, sistemas del Noguera Ribagorzana y Noguera Pallaresa, fundamentalmente). No obstante, durante el proceso de llenado, los gestores aprovechan las avenidas para incrementar más rápidamente el volumen embalsado, produciendo un claro efecto de laminación hacia aguas abajo. Además, los embalses están obligados a mantener un 
margen de seguridad que les permita mitigar la intensidad de las avenidas en cualquier época del año.

Este trabajo ha estudiado algunos aspectos generales de la laminación de avenidas en el Pirineo Central Español. Como cabría esperar, ha sido imposible identificar un patrón común a todos los embalses en su respuesta frente a las avenidas. Las conclusiones más destacadas son las siguientes:

(i) La ratio de retención explica buena parte de la capacidad de regulación de avenidas, especialmente en los embalses destinados sólo a la producción hidroeléctrica. Así, los embalses de Mediano, Canelles y Santa Ana contribuyen a reducir claramente los picos de avenida. En conjunto, el sistema de embalses del río Noguera Ribagorzana es el que produce una mayor laminación de las crecidas debido a su elevada ratio de retención.

(ii) La diferenciación de los embalses en función del uso principal del agua mejora mucho la explicación de la reducción de los picos de caudal aguas abajo. Los embalses destinados a regadio se comportan de forma menos rotunda que los hidroeléctricos. Es decir, su capacidad de reducción de los picos de crecida es inferior a la que cabría esperar de acuerdo con su ratio de retención. La razón es clara: todos los embalses para riego deben alcanzar su máximo volumen en la segunda mitad de la primavera, lo que reduce la posibilidad de laminar las avenidas de mayo a julio.

(iii) Que el patrón de gestión del embalse es una cuestión fundamental, se confirma si se considera el nivel de embalsado. Así, cuando el nivel de reserva es inferior al $70 \%$ la mayor parte de los embalses reducen dráticamente el pico de avenida (en especial, Yesa, Lanuza, Búbal, Mediano, Escales, Santa Ana y Tremp), incluso si se trata de crecidas correspondientes a un periodo de retorno de 50 años. En el caso de que el nivel de embalsado sea superior al $70 \%$ los únicos embalses que laminan la crecida de forma consistente son Mediano, Santa Ana y Tremp, y de forma más secundaria, Búbal. En esas condiciones, la avenida de 50 años apenas es laminada en Yesa ( $6 \%$ de reducción), Canelles (11\%) y Barasona (4\%), mientras los demás embalses producen aguas abajo un pico similar al de las entradas.

\section{Agradecimientos}

Este trabajo se ha realizado con el apoyo de los siguientes proyectos de investigación: "Water resources management in a changing environment; The impact of sediment on sustainability"-WARMICE (ENV-CT98-0789), "Debrisfall assesment in mountain catchments for local end users"- DAMOCLES (EVG1-1999-00027P), financiados 
por la Comisión Europea, y "Efectos hidrológicos de los cambios de uso del suelo a diferentes escalas espaciales" - HIDROESCALA (REN2000-1709-C04-01/GLO) y "Procesos hidrológicos en áreas seminaturales mediterráneas"-PROHISEM (REN20012268-CO2-01/HID), financiados por la CICYT.

\section{Bibliografía}

Adams, W.M. (1985): The downstream impacts of dam construction: a case study from Nigeria. Trans. Inst. Br. Geogr. 10, p. 292-300.

Anders Brandt, S. (2000): Classification of geomorphological effects downstream of dams. Catena, 40, p. 375-401.

Andrews, E.D. (1986): Downstream effects of Flaming Gorge Reservoir on the Green River, Colorado and Utah. Geological Society of American Bulletin, 97, p. 1012-1023.

Beguería, S. (2002): Revisión de métodos paramétricos para la estimación de probabilidad de recurrencia de eventos extremos en climatología e hidrología: El uso de series de excedencia y su comparación con las series de máximos anuales. En Cuadrat, J.M., Vicente, S.M. y Saz, M.A., eds. La información climática como berramienta de gestión ambiental. Zaragoza, Universidad de Zaragoza, p. 83-92.

Cunnane, C. (1973): A particular comparison of annual maxima and partial duration serios methods of flood frequency prediction. Journal of Hydrology, 18, p. 257-271.

García Ruiz, J.M., Puigdefábregas, J. y Creus, J. (1980): Influencia de las características físicas de las cuencas hidrográficas en la frecuencia e intensidad de crecidas. Cuadernos de Investigación Geográfica, 6, p. 19-36.
García Ruiz, J.M., Puigdefabregas, J. y Martín Ranz, M.C. (1983): Diferencias espaciales en la respuesta hidrológica a las precipitaciones torrenciales de noviembre de 1982 en el Pirineo Central. Estudios Geográficos, 170-171, p. 291-310.

García Ruiz, J.M., Puigdefábregas, J. y Creus, J. (1985): Los recursos bidricos superficiales del Alto Aragón. Huesca, Instituto de Estudios Altoaragoneses.

García Ruiz, J.M., Begueria, S., López-Moreno, J.I., Lorente, A. y Seeger, M. (2001): LoS recursos bidricos superficiales del Pirineo aragonés y su evolución reciente. Logroño, Geoforma.

Higgs, G. y Petts, G. (1988): Hydrological changes and river regulation in the UK. Regulated Rivers: Research and Management, 2, p. 349-368.

Hosking, J.R.M. (1990): L-moments: analysis and estimation of distributions using linear combinations of order statistics. Journal of the Royal Statistical Society. Serie B, Vol. 52, p. 105-124.

Greenwood, J.A., Landwehr, J.M., Matalas, N.C. y Wallis, J.R. (1979): Probability weighted moments: definition and relation to parameters of several distributions expressible in inverse form. Water Resources Research, 15, p. 1049-1054. 
López Moreno, J.I. (2002): Efecto bidrológico de los embalses en los Pirineos centrales españoles. Zaragoza, Universidad de Zaragoza. Trabajo de investigación para la obtención del Diploma de Estudios Avanzados (D.E.A.).

López Moreno, J.I., Beguería, S. y García Ruiz, J.M. (2002): Influence of the Yesa reservoirs on floods of the Aragón River, central Spanish Pyrenees. Hydrology and Earth System Sciences, 6 (4), p. 753-762.

López Moreno, J.I., Beguería, S. y García Ruiz, J.M. y Valero Garcés, B. (En prensa): Intensidad de avenidas $\mathrm{y}$ aterramiento de embalses en el Pirineo Central español. Ería.

López Moreno, J.I., Beguería, S. y García Ruiz, J.M. (2000): El régimen del embalse de Yesa (Cuenca Alta del río Aragón, Pirineo Central) y su adaptación a la variabilidad del régimen fluvial. Cuadernos de Investigación Geográfica 16, p. 131-145.

Madsen, H., Pearson, C.P. y Rosbjerg, D. (1997): Comparison of annual maximum series and partial duration methods for modelling extreme hydrologic events .1. At- site modeling. Water Resources Research, 33 (4), p. 759-769.

Martí Bono, C. y Puigdefábregas, J. (1983): Consecuencias geomorfológicas de las lluvias de 1982 en las cabeceras de algunos valles pirenaicos. Estudios Geográficos, $170-171$, p. $275-290$.

Pircher, W. (1990): The contribution of hydropower reservoirs to flood control in the Austrian Alps. In: Hydrology in Mountainous
Regions. II Artificial Reservoirs: Water and Slopes., LAHS Publ., 194, p. 3-10.

Rao, A.R. y Hamed, K.H. (2000): Flood frequency analysis. CRC Press, Boca Raton (Florida, E.E.U.U.).

Sammut, J. y Erskine, D. (1995): Hydrological impacts of flow regulation associated with the Upper Nepean water scheme, NSW. Austarlian Geographer. 26 (1), p. 71-86.

Takeuchi, K. (1998): Current status of reservoirs: Number, size and shape of reservoirs. En Takeuchi, M., Hamlin, Z.W., Kundzewicz, D. Rosbjerg \& S.P. Simonovic, eds., Sustainable reservoir development and management, IAHS Publ., 251, p. 4-7.

Thomas, D.H.L. (1996): Dam construction and ecological change in the riparian forest of the Hadejia- Jama'are floodplain, Nigeria. Land Degradation $\hat{\sigma}$ Development. 7, p. 279-295.

Vogel, R.M. y Fennessey, N.M. (1993): Lmoment diagrams should replace product moment diagrams. Water Resources Researcb. 29 (6), p. 1745-1752.

White, S., García Ruiz, J.M., Martí-Bono, C., Valero, B., Errea, M.P. y Gómez-Villar, A. (1997): The Biescas campsite disaster and its temporal and spatial context. Hydrological Processes, 11, p. 1797-1812.

Zsuffa, I. (1999): Impact of Austrian hydropower plants on the flood control safety of the Hungarian Danube reach. Hydrological Sciences Journal, 44 (3), p. 363-371. 